\title{
ESTRATEGIAS DE EDUCACIÓN PARA LA PAZ: UNA MIRADA DESDE SU APLICACIÓN EN COMUNIDADES VÍCTIMAS DE LA VIOLENCIA FRENTE A LA ARTICULACIÓN DE LA INSTITUCIONALIDAD EN LOS MONTES DE MARÍA
}

\author{
Sandra Milena Márquez Cárdenas ${ }^{1}$ \\ Georgina Isabel de León Vargas ${ }^{2}$ \\ Wilson Yesid Suárez Manrique ${ }^{3}$
}

\section{Resumen}

En este artículo se analizan los aportes de las estrategias de educación para la paz, en comunidades víctimas de la violencia armada en Montes de María. El análisis se aborda desde el estudio de caso, producto de la aplicación metodológica de estrategias de educación para la paz en comunidades víctimas de la violencia, permitiendo inferir la necesidad de la educación para la paz en estas, y deja en evidencia los retos en la aplicación de la educación para la paz en comunidades con características sociales, culturales, económicas, similares. Asimismo, se presenta el análisis de los resultados desde la aplicación de estrategias pedagógicas para la paz y su aprehensión en comunidades víctimas de la violencia, observando cambios significativos, que permiten el análisis de la transformación

1 Magister en Conflicto y Paz. Especialista en Derechos Humanos y Derecho Internacional Humanitario. Docente Investigadora. Grupo GISCER. Asesora Jurídica Centro de Orientación Sociojurídica a víctimas del conflicto -CECAR. Email: sandra.marquez@cecar.edu.co.

2 Abogada, Magíster en Derecho Laboral Universidad Autónoma de Guerrero México, Docente Tiempo completo Corporación Universitaria Rafael Núñez, Coordinadora de Investigación Programa de Derecho, sede Cartagena Corporación Universitaria Rafael Núñez, Integrante del Grupo de Investigación Derecho Público Categoría C COLCIENCIAS de la Corporación Universitaria Rafael Núñez. Email: georgina.deleon@curnvirtual.edu.co

3 Doctor en Derecho de la Universidad de Salamanca. Profesor investigador de la Corporación Universitaria Rafael Núñez sede Cartagena, integrante del Grupo de investigación Derecho Público de la Corporación Universitaria Rafael Núñez sede Cartagena. Dirección postal Calle 200 N 14-50, Colombia, código postal 681004. Email: wilsonyesidsuarez@gmail.com 
no violenta del conflicto. Si bien hay antecedentes de aplicación de estrategias pedagógicas para la paz, su aprehensión en las comunidades victimizadas por la violencia, han demostrado la flexibilidad de las mismas y su base en el aprendizaje desde la cotidianidad de las comunidades. En los hallazgos encontramos la importancia de la empresa privada en el territorio como actor estratégico de desarrollo, los retos para la construcción de paz territorial y la flexibilidad imperativa de las estrategias de educación para la paz.

Palabras Clave: Educación para la paz, comunidades víctimas, conflicto social, Montes de María.

\begin{abstract}
In this article we analyze the contributions of peace education strategies in communities victims of armed violence in Montes de María. The analysis is approached from the case study, product of the methodological application of peace education strategies in communities victims of violence, allowing to infer the need for peace education in these, and exposes the challenges in the application of education for peace in communities with social, cultural, economic, similar characteristics. Likewise, the analysis of the results is presented from the application of pedagogical strategies for peace and their apprehension in communities victims of violence, observing significant changes that allow the analysis of the non-violent transformation of the conflict. Although there is a history of application of pedagogical strategies for peace, their apprehension in the communities victimized by violence has demonstrated their flexibility and their basis in learning from the daily life of the communities. In the findings we find the importance of private enterprise in the territory as a strategic development actor, the challenges for the construction of territorial peace and the imperative flexibility of education strategies for peace.
\end{abstract}

Keywords: Education for peace, victim communities, social conflict, Montes de Maria.

\title{
Introducción
}

econstruir el tejido social de una comunidad víctima de la violencia
es un proceso que inicia con la participación de todos los actores,
redes y mesas de la región, incluyendo, el Estado, los medios de
comunicación, las empresas privadas, los organismos de cooperación in-
ternacional, y la sociedad civil y sus formas de organización. En la región
de Montes de María, se han dado avances en cuanto al trabajo en red y el
empoderamiento de líderes y lideresas de las comunidades rurales donde 
se vivieron las más nefastas masacres, como una forma de motivar los procesos sociales y construir puntos de encuentro entre semejantes. Con el objetivo de analizar la contribución de la educación para la paz, implementada en comunidades rurales victimizadas de la subregión de los Montes de María. Se presenta una aproximación de la estrategia metodológica de educación para la paz: Reconstruyendo el tejido social: una apuesta a la participación comunitaria, desarrollada en el interior de las comunidades, y dirigida a la superación de conflictividades de estas. Los capítulos de este escrito, analizan los contextos históricos de los municipios que integran la subregión de los Montes de María. Se relacionan los puntos clave de la aprehensión de las estrategias utilizadas en las comunidades desde la cooperación de la empresa privada.

Analizar los programas Colombia en paz y Colombia equitativa y sin pobreza extrema del Plan Nacional de Desarrollo Todos por un nuevo país 2014-2018, a la luz de la teoría del desarrollo de Amartya Sen, frente a la materialización de las apuestas políticas en los municipios de San Juan Nepomuceno y el Carmen de Bolívar se convirtió en el objetivo general del proyecto de investigación que le da vida al presente escrito. Se aborda conceptualmente el plan nacional de desarrollo- PND, la teoría del desarrollo, la pobreza extrema, la equidad y la construcción de paz en Colombia, analizando cómo estas categorías se materializan en las realidades sociales y políticas de los municipios de San Juan Nepomuceno, el Carmen de Bolívar siendo estos golpeados igualmente por el conflicto armado, pero receptores de dos niveles distintos de intervención estatal.

El principal objetivo es conocer cómo se construye la paz (De León Vargas, G. I., Ballestas León, D. M., \& Martínez Martínez, F. (2017), en cada uno de ellos y hasta qué medida la pobreza es limitante de esta. Carmen de Bolívar territorio modelo y pionero en aceptar que la paz es una necesidad de todos para garantizar la no repetición de los hechos de barbarie que hicieron que el territorio se visibilizarla de forma negativa en el todo el país por ello la labor de todos los pobladores consistió en visibilizarlo de manera positiva encarando la paz desde adentro tejida por todos y cada uno de los sobrevivientes del conflicto líderes sociales, desplazados políticos, profesores, periodistas, mujeres, campesinos, y sindicalistas los cuales en un espacio de convivencia se reúnen cada primer sábado del mes 
a analizar la situación del territorio y ofrecen estrategias para la reconstrucción de su tejido social de la esperanza y de la reconciliación, cabe destacar que adentrarse al territorio es evidenciar que saltan a la vista flagelo de desigualdad pobreza extrema que se convierten en un limitante para la verdadera y duradera paz que se desea por todos los que fueron testigos del horror de la guerra y los que ahora son tejedores de paz.

Finalmente, en el análisis de los resultados podremos observar derroteros, para posteriores estudios en los municipios, de cara a planes, y/o proyectos locales de reconstrucción del tejido social desde los postulados de la educación para la paz. Se caracterizan las estrategias abordadas en concordancia con la educación para la paz, producto del resultado del diagnóstico participativo de corte socioeconómico, realizado desde las comunidades victimizadas por la violencia de los Municipios de San Onofre, El Carmen de Bolívar, Toluviejo y Ovejas.

En términos generales, se analiza la contribución de la educación para la paz en la transformación no violenta del conflicto en estas comunidades rurales y los retos que en la actualidad tiene su aplicación en comunidades con características similares, como la resistencia a la violencia armada, la presencia de la empresa privada en el territorio, los conflictos comunitarios, la ingobernabilidad, la poca participación social y la necesidad del trabajo en red.

De acuerdo con Jean Paul Lederach (2000), la transformación de conflictos en comunidades víctimas de la violencia implica percibir y responder a fluctuaciones de los conflictos sociales como una oportunidad para crear procesos de cambio constructivos que reduzcan la violencia. Así desde la pedagogía para la paz, en comunidades que han pasado episodios largos de violencia armada, se convierte en eje fundante de una mejor convivencia y en preparación de escenarios de desarrollo territorial desde distintos ejes: políticos, sociales, culturales, económicos y de goce efectivo de derechos.

La flexibilidad de estrategias pedagógicas para la paz, debe contribuir al aprendizaje desde los saberes intrínsecos de las personas que comparten un horizonte de futuro, garantizando que la aprehensión de los conocimientos básicos propuestos se pueda desarrollar respetando el auto proceso de aprendizaje. 
El monitoreo y revisión constante de las estrategias de educación para la paz implica el aprendizaje de ser miembros de una comunidad global, en el sentido de la convivencia armoniosa y la resolución pacífica de conflictos, sentar estas bases significa preparar a las nuevas generaciones y aportar a los espacios de encuentros culturales que para comunidades víctimas en Montes de María ha sido la manera más fehaciente de resistencia.

\section{Metodología}

La metodología que se utilizó es un estudio de casos, de tipo explicativo- experimental, puesto que se adentró en terreno para explicar los fenóme- nos que ocurren en la realidad, así como determinar su origen y causas. Desde lo experimental, es posible plantear la causa-efecto de la problemá- tica. Es de carácter socio-jurídica, puesto que interpela "los espacios de jurídica, los saberes jurídicos concretos y la práctica jurídica" (Hernández S., Fernández C., Baptista L., 2010). Esta puede entonces crear derecho, regular fenómenos culturales que son relevantes para un Estado en un momento histórico determinado". En este sentido está basada en un estu- dio descriptivo, puesto que su principal objetivo es el análisis de la relación de las propuestas territoriales para la construcción de paz y la erradicación de la pobreza extrema con el PND, y de esta forma diagnosticar el estado de los municipios de San Juan Nepomuceno y el Carmen de Bolívar.

El método que se implementó fue el diseño de campo, por medio del cual se analizaron las situaciones que se presentan en los territorios. En este sentido se utilizaron técnicas de recolección de información como la observación, retomada como una estrategia fundamental del enfoque cualitativo, pero a su vez como un proceso riguroso de investigación, que permite describir situaciones y/o contrastar hipótesis, siendo por tanto un método científico. Esta técnica será orientada a la respuesta concreta de los objetivos específicos. Por tanto, para efectos de esta investigación, será intencionada, selectiva e interpretativa y semi-estructurada, es decir, contará con una guía previamente elaborada que permitirá dar paso o tener acceso a cualquier otra información relacionada con el problema de investigación.

La entrevista como "la comunicación interpersonal establecida entre el investigador y el sujeto de estudio a fin de obtener respuestas verbales a 
los interrogantes planteados sobre el problema propuesto" (Canales Cerón, 2006). Se considera que este método es más eficaz que el cuestionario, ya que permite obtener una información más completa. Esta también será semi-estructurada, con el ánimo de permitir que los sujetos puedan realizar intervenciones de acuerdo a su perspectiva de la materialización del PND en el territorio.

El proyecto de investigación se llevó a cabo en bajo el enfoque cualitativo, ya que se desarrolló a partir de instrumentos y técnicas de este enfoque. Jurídicamente se ubica en investigación aplicada, puesto que incursionará entre la relación del Derecho y el Estado colombiano, a partir del sentido político que tiene la juridicidad en los municipios mencionados anteriormente, con el fin de generar propuestas encaminadas a la transformación social y a la superación del estado de pobreza extrema.

\section{Contexto de violencia en Montes de María}

Los innumerables conflictos sociales, que han rodeado la historia de Co- lombia han trascendido todas las esferas, particularmente en los entornos rurales del país. Se entiende por conflicto social el fenómeno presente en la historia de la humanidad y sus consecuencias en el desarrollo humano.

Colombia, según Héctor Alfredo Salazar (2014), ha sufrido numerosos conflictos sociales violentos. Desde las sociedades de chibchas, taironas, quimbayas, calima, motilones y otros hasta la llegada de los españoles y el sometimiento del indígena a la esclavitud se vivieron momentos de fuertes enfrentamientos. La mezcla de razas en lo que hoy es Colombia y la búsqueda de la formación del estado-nación estuvieron rodeadas de episodios conflictivos, no solo vistos desde la violencia armada sino desde otras esferas sociales como el predominio del patriarcado, el papel de la iglesia, la progresividad de la protección de los derechos humanos, entre otros.

El Estado colombiano siempre se ha caracterizado por las confrontaciones violentas en esa búsqueda insaciable de consolidación de una nación. Muestra de ello son las múltiples constituciones políticas que demarcan el dominio de poder de la clase política del momento, el cual genera elementos para analizar la violencia estructural. Esta es entendida desde la tesis del profesor Johan Galtug (2004) como una estructura demasiado 
represiva, explotadora o alienante, demasiado estricta o permisiva para la comunidad del pueblo.

Colombia en su división político-administrativa, está compuesta por treinta y dos departamentos y cinco distritos (Departamento Administrativo Nacional de Estadística, 2005) (Colombia.co, 2013). Los departamentos son las unidades territoriales de primer nivel en el país, gobernados desde sus respectivas ciudades capitales. Estos departamentos forman regiones con características similares, entre estas, culturales, económicas y sociales, y gozan de autonomía administrativa (Constitución Política de Colombia, 1991, art. 289). Los departamentos están integrados en su geografía política por municipios, los cuales corresponden al segundo nivel de división administrativa en Colombia, que mediante agrupación conforman los departamentos. Colombia posee 1.122 municipios registrados ante el DANE- Departamento Administrativo Nacional de Estadísticas que de acuerdo con el Artículo 311 de la Constitución Política de Colombia de 1991, la Ley 136 del 2 de junio de 1994 y la ley 1551 de 2012 es la entidad territorial fundamental de la división político-administrativa del Estado, con autonomía política, fiscal y administrativa dentro de los límites que le señalen la Constitución y las leyes de la República.

La Oficina de Estudios Geográficos del Instituto Geográfico- Agustín Codazzi establece que el departamento de Sucre es uno de los treinta y dos departamentos que forman la República de Colombia. Su capital es Sincelejo, ubicado al norte del país, en la región Caribe. Se divide en veintiséis municipios y cinco subregiones naturales: La Mojana, Montes de María, Morrosquillo, Sabanas y San Jorge.

La subregión natural de Los Montes de María está compuesta por quince municipios de dos departamentos: ocho de Sucre; Chalán, Toluviejo, San Antonio de Palmito, Coloso, San Onofre, Los Palmitos, Morroa y Ovejas y siete de Bolívar; El Carmen de Bolívar, San Juan Nepomuceno, Córdoba, Zambrano, María La Baja, San Jacinto, El Guamo.

Los Montes de María han sido históricamente un territorio de grandes riquezas naturales y abundancia de alimentos, hasta el punto de ser considerada La despensa del Caribe. De gran producción ganadera, poblada prin- 
cipalmente por familias campesinas, afrocolombianas e indígenas, que encontraron allí tierras baldías y formaron asentamientos después del régimen de la esclavitud que azotó a colombianos (Mendoza, 2010, pág. 7).

Presenta una movilización masiva del campo, zona rural, a la ciudad por la presencia de los actores armados en el territorio, siendo su punto más álgido entre los años 1993 y 2005. La conflictividad en Montes de María tiene múltiples miradas. Una de ellas es sobre la tenencia de la tierra, que en Colombia y en especial en las zonas rurales está vinculada al dominio y poder territorial, y a la acción de los actores armados.

La historia de poblamiento de Montes de María y sus rasgos culturales constituyen factores determinantes en el desarrollo del conflicto e igualmente en el anhelo de paz (PNUD, 2010). En los años 90, según la documentación del Centro Nacional de Memoria Histórica - CNMH- llegan al territorio de Montes de María grupos guerrilleros que se acentuaron en la zona y comenzaron a extorsionar y secuestrar finqueros y terratenientes, muchos de los cuales malvendieron sus tierras por el temor. (Grupo de Memoria Historica. GMH, 2013)

Las FARC E.P llegaron a Montes de María a inicios de la década de los noventa, luego de las desmovilizaciones de otras guerrillas anteriormente asentadas en la región (Corriente de Renovación Socialista - CRS - Partido Revolucionario de los Trabajadores PRT -); sin embargo, su accionar inició drásticamente en 1994 y se mantuvo con índices elevados hasta 2003. Esta organización guerrillera hizo presencia en la subregión a través de los frentes 35 y 37, adscritos al Bloque Caribe, que no son oriundos de la región sino desplegados del Frente 18, que operaba en Córdoba y el Bajo Cauca. En la zona de montaña se ubicó el Frente 35, donde convergió con estructuras del ELN y el ERP, y en las riberas del río Magdalena operó a través del Frente 37. Paralelamente se agudiza el conflicto por fraudes electorales, la corrupción y el control estratégico del territorio por grupos dedicados al narcotráfico. (pág. 128)

También es importante mencionar la llegada de las llamadas cooperativas de vigilancia y seguridad privada - Convivir - de los municipios de San 
Onofre y Coveñas - Sucre, las cuales mutan posteriormente a las Autodefensas - ACCU.

Los actos terroristas de los grupos armados ilegales, llámenseparamilitares o guerrillas, azotaron la región con masacres, desapariciones, desplazamiento forzado, homicidios selectivos, vinculación de menores a grupos armados al margen de la ley, minas antipersonales, bombas y secuestros. Adicionalmente, el Estado se vio involucrado en los desmanes de violencia que vivieron los Montes de María con asesinatos selectivos ejecutados en complicidad con estructuras paramilitares de la zona. Así lo muestra la línea de tiempo elaborada por el Colectivo de Comunicaciones Montes de María línea 21 para el Museo Itinerante de la Memoria y la Identidad de los Montes de María (MIM):

Incursión sistemática de policías disfrazados de paramilitares. Con las primeras células de policías que de día iban como el Cuerpo Técnico de Investigación - CTI - y por la noche se ponían pasamontañas de limpieza y a matar gente y a todos los que contradecían los mataban y los desaparecían.

Otra de las causas estructurales del conflicto en Montes de María es la carencia de un modelo de desarrollo equitativo e incluyente. A pesar de ser considerada la despensa del Caribe, los índices de pobreza multidimensio- nal son muy elevados, debido a diversos factores como: difícil acceso a la educación, la empleabilidad, servicios de salud ineficaces, índices de desnutrición, embarazos en adolescentes, incultura política, corrupción, explotación laboral, entre otros.

Montes de María representa para Colombia la resistencia, la superación de conflictividades y la construcción de paz desde la identidad territorial, sin olvido de lo sucedido. Basta con analizar la dinámica del conflicto armado esbozado y sus horrores en el territorio para dimensionar el terror que caracterizó esos tiempos y en particular las consecuencias palpables en la población rural.

En la subregión de Montes de María, en respuesta a la resistencia se fueron formando movimientos sociales que impulsan propuestas de re- 
construcción regional direccionadas al desarrollo agrario, social y sostenible. Estas propuestas conllevan el compromiso de los distintos sectores de la sociedad a involucrarse activamente en la construcción conjunta de soluciones, así como el cumplimiento de grandes retos económicos, sociales y competitivos que hoy por hoy presentan las zonas rurales, como una forma de reconstrucción de sociedad.

En el proceso de recuperación del territorio, para hacer frente a la violencia en los Montes de María, inicio su intervención con el aumento del pie de fuerza pública y militar, sin embargo hubo consecuencias nefastas a esta incidencia; se genera así un cambio no tan profundo en la dinámica del conflicto armado, lo que conlleva a re pensar en las estrategias de intervención en las comunidades victimizadas.

La confluencia de varios actores armados, la constante ingobernabilidad y la marginación histórica de la región representa para la zona rural obstáculos de desarrollo social y sostenible de sus comunidades.

La pobreza, la ausencia del Estado y la crisis de gobernabilidad que atraviesa el territorio montemariano siguen siendo realidades visibles que ameritan una mirada permanente y cuidadosa, y al tiempo una intervención constante de organismos y entidades que articuladamente promuevan acciones a favor del desarrollo y la paz.

Según la Red Nacional de Información (Unidad para las Víctimas, 2018), el departamento de Sucre registra 300.865 víctimas del conflicto armado. Entre los años 2000 y 2002 se presenta la mayor cantidad de declaraciones de las personas que sufrieron los hechos violentos que cercenan sus derechos humanos. Esta cifra implica para el Estado respuestas eficaces y efectivas para la superación de las condiciones de vulnerabilidad, posteriores a la ocurrencia de los hechos victimizantes.

\section{Hacia la implementación de estrategias de educación para la paz}

Fortalecer la reconstrucción del tejido social en una zona tan devastada por la violencia requiere el despliegue de acciones conjuntas que transformen el conflicto social en clave de no violencia. La consecución de una ciudadanía educada y una verdadera sociedad civil con un imaginario de región, un proyecto colectivo de futuro con sentido de pertenencia, en el 
que cada cual tenga claro sus deberes y derechos ciudadanos, se convierte entonces en el punto clave de la formación que permita participar a la comunidad en la toma de las decisiones que les competen, así como también en respuestas estratégicas a las conflictividades planteadas en esta restauración social.

Educar para la paz en contextos de conflicto implica educar sobre el conflicto, que no debe ser confundido con la violencia, atendiendo a que la paz, en teoría de Johan Galtung en su texto Pazpor medios pacíficos (2003), es "la fase superior de los conflictos", es decir, el estadio en que los conflictos son transformados por los pobladores de la comunidad - territorio de forma positiva, creativa y no violenta. Esta teoría ha sido implementada desde los postulados de la cultura de paz, que para los Montes de María se ven reflejados en la construcción de redes y bases de apoyo entre comunidades rurales.

El concepto contemporáneo de educación tiene orígenes filosóficos antiguos y hoy los interesados por la educación para la paz tienen una deuda "considerable con las ideas cosmopolitas y con las preocupaciones éticas por la paz de siglos anteriores” (Hicks, 1993, pág. 38). Surge en los años 50 el campo de investigación sobre la paz, el cual contempla cómo la violencia estructural puede conducir a la muerte o a una disminución del bienestar y del potencial humano, a consecuencia de varios factores, entre estos las diferentes conflictividades que rodean el ser humano. Vicenc Fisas (2011), en sus escritos de educar para una cultura de paz, propone la transformación del conflicto desde una esfera creativa y no violenta. Para ello resulta fundamental estimular la creatividad, donde prevalezca la comprensión mutua, la tolerancia y el desbloqueo de posiciones, sobre todo en comunidades históricamente victimizadas por factores de violencia.

La educación es, sin duda alguna, un instrumento crucial de la transformación social y política. Si estamos de acuerdo en que la paz es también la transformación creativa de los conflictos, y que algunas de sus palabras-clave son el conocimiento, la imaginación, la compasión, el diálogo, la solidaridad, la integración, la participación y la empatía, hemos de convenir que su propósito no es otro que formar una cultura de paz, opuesta a la cultura de 
la violencia, que pueda desarrollar esos valores, necesidades y potencialidades. Es a través de la educación "que podremos introducir de forma generalizada los valores, herramientas y conocimientos que forman las bases del respeto hacia la paz, los derechos humanos y la democracia, porque la educación es un importante medio para eliminar la sospecha, la ignorancia, los estereotipos, las imágenes de enemigo y, al mismo tiempo, promover los ideales de paz, tolerancia y no violencia, (Fisas, 2011, p. 374).

La reconstrucción del tejido social desde la educación para la paz en una comunidad es un proceso que inicia con la participación de todos los ac- tores, redes y mesas de la región, incluyendo el Estado, los medios de co- municación, las empresas privadas, los organismos de cooperación inter- nacional y la sociedad civil y sus formas de organización. En la región de Montes de María se han dado avances en cuanto al trabajo en red y empo- deramiento de líderes y lideresas de las comunidades rurales donde se vi- vieron las más nefastas masacres, como una forma de motivar los procesos sociales y encontrar puntos de encuentro entre semejantes. Así, John Paul Lederach, en su escrito La paz a escala popular, propone la capacidad de alcanzar consensos desde las acciones comunitarias propias del diario vivir (Lederach, 2000, p. 15).

La paz a escala popular (Lederach, 2000) describe el reconocimiento de la paz como concepto, el cual tiene la gran capacidad cualitativa de expresar un ideal y una ilusión humana, y entiende lo popular como uso sistemático de las relaciones interpersonales. Si bien se plantea desde un ideal, no es menos cierto que en la educación para la paz en comunidades rurales, victimizadas por la violencia armada, con características como las de Montes de María, resulta viable comprender el concepto desde su afirmación ideal de convivencia deseable, teniendo claro que la cultura y la constitución como vereda incide directamente en la aprehensión de conocimientos de educación para la paz.

La Fundación Red Desarrollo y Paz de los Montes de María, en su rol de orientador político y estratega del Programa de Desarrollo y Paz de los Montes de María - PDP Montes de María -, promueve en el territorio un con- 
junto de iniciativas construidas y protagonizadas por los pobladores, concertadas con las comunidades victimizadas de la región y en coordinación con la academia desde el liderazgo de la incidencia de la Corporación Universitaria del Caribe - CECAR - desde su visión y misión regional. En este contexto todos los procesos susceptibles de intervención tienen un horizonte de gran impacto y se orientan hacia la transformación no violenta de las realidades sociales, políticas, económicas y culturales que interpelan constante y permanentemente las acciones trascendentales dirigidas a las personas participantes de los encuentros pedagógicos, reflejadas en los resultados y en el entorno familiar, comunitario, laboral y de goce efectivo de derechos.

El imaginario de región se construye desde los siguientes ejes estratégicos de los postulados de educación para la paz:

1. Eje estratégico construcción de lo público: Cualifica y moviliza la participación ciudadana para la incidencia en políticas públicas a nivel local, regional y nacional. Presenta acciones de fortalecimiento a la institucionalidad pública de Montes de María y genera espacios de interlocución entre la institucionalidad y la sociedad civil.

2. Eje estratégico desarrollo socioeconómico sostenible y sustentable: Contribuye desde sus acciones a las relaciones sociales y económicas incluyentes, justas, solidarias y equitativas.

3. Eje estratégico reconstrucción del tejido social: Impulsa de manera permanente la participación en la construcción de una visión regional de desarrollo humano integral y facilita la formación y el fortalecimiento de organizaciones y redes sociales, acompañándolos mediante un modelo pedagógico integral.

4. Eje estratégico de derechos humanos, paz y reconciliación: Busca la promoción de una cultura basada en el marco ético de los derechos humanos, que favorece la prevención y la transformación no violenta y creativa de los conflictos, facilita procesos de reconciliación y de justicia restaurativa. 
En este sentido, en la zona rural de los municipios de Ovejas y San Ono- fre- Sucre y de El Carmen de Bolívar- Bolívar se aplicaron estrategias pe- dagógicas para la paz en el fortalecimiento comunitario entre 2012 y 2014, en 2015 se aplicaron en las comunidades rurales de Toluviejo Sucre desde acciones articuladas en el proceso estratégico territorial - PET Montes de María 2012 - 2014, para efectos de desarrollo conceptual, según el Pro- grama de Desarrollo y Paz, se denomina reconstrucción del tejido social. Estas acciones estratégicas, acompañadas de un modelo pedagógico ba- sado en la cultura de paz, han permitido la participación permanente en la construcción de políticas públicas que permiten el goce efectivo de los derechos humanos.

$\mathrm{El}$ argumento de la cultura de paz en la óptica educativa pasa por educar en y para el conflicto. Por esta razón, resulta fundamental estimular la creatividad, para que al buscar soluciones a los conflictos prevalezca la comprensión mutua. Igualmente se tiene en cuenta el lenguaje y las premisas de educación para la paz en sentido comunitario, como empoderamiento de los pobladores y transformación no violenta del conflicto.

Paulo Freire consiguió construir un cuerpo teórico y una pedagogía volcada a la práctica, a la acción transformadora. El autor afirma en Pedagogía del oprimido (1975) que: "la mejor manera de pensar es pensar en la práctica" y generar una pedagogía posicional, rechazando la idea de neutralidad. Él planteaba, con mucha insistencia, que la neutralidad no es posible en el arte educativo ni en el acto educativo. Comprendido este en la educación para la paz, desde el aprendizaje en la comunidad y su participación activa en los procesos de formación.

La pedagogía de la educación popular tiene un sentido netamente transformador. Este cambio para el estudio en mención constituye la base conceptual, ya que considera la importancia de empoderar pacíficamente a comunidades victimizadas por la violencia, teniendo en cuenta sus vivencias y características sociales, culturales y de convivencia comunitaria así como las consecuencias palpables en el territorio y en el proyecto de vida de los lugareños.

Freire propugnó desde el momento en que construyó su método de alfabetización que el dominio de la palabra, saber escribir y saber leer, solamente tienen sentido si se traduce en una mejor lectura del mundo, una 
mejor lectura del contexto del ser humano. El autor plantea una tolerancia que significa diálogo, una actitud fundamental que estaba presente en toda su práctica cotidiana, y relaciona de esta manera sus postulados con la pedagogía esperanzadora: ambas sientan los preceptos de aplicación y contribución de educación para la paz.

Las contribuciones de Freire a la pedagogía para la paz, según Eduardo Andrés Sandoval Forero en la Revista de Pazy Conflictos (2015), se basan fundamentalmente en los siguientes postulados:

- Educar es conocer críticamente la realidad.

- $\quad$ Educar es transformar la realidad.

- Educar es formar sujetos de cambio.

- Educar es diálogo.

- Educar no es un acto individual ni intelectual.

- Educar es un proceso colectivo y práctico.

- $\quad$ Educar involucra diferentes formas de saber: la conciencia, el sentimiento, el deseo y la voluntad.

- Educar es generar experiencias colectivas y dialógicas entre educandos y educadores.

- Educar es construir nuevos conocimientos entre educandos y educadores.

- $\quad$ Educar es conocer el mundo desde y en la práctica transformadora.

- $\quad$ Educar es el proceso en el cual intervienen valores, voluntades, deseos, utopías, emociones e imaginación.

Este aporte contribuye esencialmente a las estrategias de educación para la paz basada en los conocimientos autóctonos de la comunidad en la que se aplican. De esta manera, se consolidan desde los sectores populares he- rramientas educativas que les permiten organizarse y transformar sus en- tornos a través de sus propias vivencias, con el fin de mejorar sus vidas. 


\section{La responsabilidad social empresarial en la reconstrucción del te- jido social}

En revisión documental, sobre la presencia de la empresa privada en el territorio, se ha podido constatar que, teniendo en cuenta la presencia la empresa privada en el territorio de Montes de María, el grupo Argos, en el marco de la responsabilidad social empresarial - RSE - de la filial Argos Cemento, realiza convenio con la Fundación Red Desarrollo y Paz de los Montes de María en los años 2013, 2014 y 2015. Priorizando las zonas rurales de los Municipios de San Onofre, El Carmen de Bolívar, Ovejas y Toluviejo, en el entendido que en estas zonas tendría su área de influencia la empresa.

El convenio gira en dos ejes fundamentales:

1. Diagnóstico socioeconómico en los municipios.

2. Proceso de fortalecimiento comunitario en los municipios y su zona rural

Estos dos ejes permiten, desde el estudio de caso en las comunidades vic- timizadas por la violencia interpretar los resultados de las estrategias de educación para la paz implementadas desde los postulados del - PDP Montes de María y sus aliados estratégicos en el territorio, entre estos la Corporación Universitaria del Caribe - CECAR - como medida de fortalecimiento comunitario y transformación no violenta del conflicto.

La Fundación Red Desarrollo y Paz de Los Montes de María, consecuente con los desafíos y potencialidades que interpelan a la región y conocedora de su rol articulador, dinamizador y facilitador, plantea en sus ejes estratégicos la orientación al fortalecimiento organizacional; la reconstrucción del tejido social; la construcción de lo público, los derechos humanos, la paz y la reconciliación; el desarrollo socioeconómico sostenible y sustentable, como los derroteros para la creación, aplicación y ajuste metodológico que harán posible avanzar hacia el empoderamiento pacífico y un buen vivir de las comunidades victimizadas. En este sentido desde el eje que se ha denominado Reconstrucción del tejido social se fortalecen los procesos de formación y articulación de la comunidad en redes de trabajo, para construir convivencia, confianza, capacidad de autogestión, identidad 
Estrategias de educación para la paz: una mirada desde su aplicación

con el territorio y condiciones que favorezcan un desarrollo comunitario en Montes de María.

\section{Eje de derechos humanos, paz y reconciliación}

Desde una ciudadanía activa, se busca la promoción de una cultura basada en el marco ético de los derechos humanos como principios de conviven- cia y paz, la transformación no violenta y creativa de los conflictos, y el fomento de espacios parala reconciliación que posibiliten una paz estable y duradera en Montes de María. Partiendo de estas premisas se crea la herramienta metodológica Reconstruyendo el tejido social: una apuesta a la parti- cipación comunitaria, la cual es una guía para el abordaje de educación para la paz en las comunidades victimizadas, que atiende a las características co- munes presentadas en Montes de María.

Reconstruyendo el tejido social: una apuesta a la participación comunitaria es una herramienta participativa que busca desarrollar una intervención enmarcada en un contexto de valoración de las identidades locales en el proceso de recuperación después de episodios de la violencia. Permite trabajar con diversos actores del territorio desde una técnica lúdica, experiencial y constructivista, donde se promueve la cohesión social, el desarrollo comunitario, la organización y movilización conjunta para la consecución de sus objetivos, y la promoción de sus capacidades de incidencia a los niveles de acción en que se requieran. Así este estudio de caso se enfoca en el empoderamiento pacifista observado en las comunidades víctimas de la violencia en 4 municipios de Montes de María, en el marco del desarrollo delos ejes contemplados en el convenio FRDPMMa - Argos.

La herramienta mencionada con antelación permite reconstruir la sociedad a partir de una propuesta de participación ciudadana y contribuye al desarrollo de capacidades que se denominan de acción consciente, por medio de las cuales la participación de los diferentes actores se combina de manera prudente en el desarrollo de la estrategia metodológica, favoreciendo la toma de conciencia de sus similitudes e intereses comunes, y las posibilidades de incrementar el potencial de operación por medio de acciones concertadas y organizadas, acciones que en la misma medida generen como subproducto sentido de pertenencia, solidaridad y capacidad de 
involucramiento en la construcción de un destino común, visión de desarrollo sostenible y sustentable en clave de la cultura para la paz.

Se espera que la comprensión verdadera como acción consciente se manifieste espontánea, natural y sencilla, en especial sea dignificante para los participantes, quienes reflejan en sus distintos hábitos la comprensión de los temas que se abordan desde la estrategia encuadrada en la educación para la paz.

\section{De la aplicación conceptual de postulados de educación para la paz a la transformación pacífica del conflicto}

La educación para la paz en comunidades víctimas de la violencia, da res- puesta a nuevas formas educativas, es decir a nuevas prácticas para abordar los contextos sociales donde hay o ha habido experiencias de conflicto y violencia, sea estructural, directa o cultural, igualmente como herramientas de prevención de futuros conflictos.

La transformación no violenta de esta realidad, la movilización social, el trabajo en redes y la articulación y formación en las comunidades rurales se facilita con postulados de educación para la paz, implica la capacitación y formación de los actores en búsqueda de un bien común, para contribuir con la potenciación de sus capacidades. En relación con la cultura depaz, la cual se entiende como un conjunto de valores que coloca en práctica la no-violencia por medio de la educación, el dialogo y la cooperación (ONU, Resolución No. 53/243, Declaración y Programa de Acción sobre una Cultura de Paz).

\section{Metodología investigación-acción participativa (IAP)}

La metodología investigación acción participativa -IAP- es una técnica particular, fruto de la reflexión, el trabajo y la experiencia, que se aplica en numerosas zonas del planeta, principalmente en América Latina y España. Presenta como rasgo definitorio la elaboración de procesos participativos abiertos e incorpora a todos los sectores de la ciudadanía en la realización de planes de desarrollo local y comunitario. La IAP no ofrece una batería de respuestas y soluciones a los problemas, sino que propicia la conversación y el diálogo como mecanismo para que los sujetos afectados aporten 
soluciones a sus problemas. Al elaborar las respuestas con todos los sectores sociales de la localidad, evidentemente, se incrementa la posibilidad de resolución pacífica del conflicto a partir de ideas que se reconocen como sentires propios de la población afectada, quien a su vez proporciona respuestas, soluciones y propuestas de acción que se ajustarán a la realidad concreta vivenciada en el meollo del conflicto, dado que han sido elaboradas y compartidas por la ciudadanía en el proceso de investigación - acción.

Para hacer operativo este enfoque se lleva a cabo la siguiente secuencia metodológica, aplicada en zonas rurales de Montes de María, como El Carmen de Bolívar, Ovejas, San Onofre y Toluviejo:

Diagnóstico: se encuentra desde el comienzo del proceso. Como primera medida se realiza una indagación sobre los problemas, demandas y autocríticas expuestas por los distintos actores que conviven en el municipio. El propósito es doble: por un lado, explicar la realidad territorial y, por otro, analizar las carencias presentes para comprender el enfoque que se le puede dar a estos problemas desde la educación para la paz. Es decir, estamos ante una valoración provisional, que tiene un carácter de síntoma o de situación problemática y que requiere la intervención de los actores sociales para reflexionar sobre sus preocupaciones. $\mathrm{Y}$ ante una investigación que conlleve acciones consecuentes, además de ser un diagnóstico objetivo de la realidad.

Análisis de redes y de conjuntos de acción: como parte de la secuencia metodológica se involucra en la investigación a todos los sectores sociales, como ciudadanos/as, empresarios/as, asociaciones formales y grupos informales, con el fin de documentar las experiencias, sentires, a partir de la posición social parcial de cada uno de ellos y de las interacciones que se producen entre sí. De esta manera, se hace posible iniciar la construcción de propuestas y acciones que permitan alcanzar los objetivos previstos. Este análisis favorece la concreción de los planes de acción y la consecución de las estrategias propuestas, incluyendo la potencialidad de cada uno de los actores, equivalente al proceso de empoderamiento desde la mirada teórica de la paz positiva, propuesta por Francisco Muñoz (Muñoz, 2001, p.3). 
Como parte fundamental del desarrollo en la observación de redes y conjuntos de acción se analizan las actitudes de los actores a partir de la identificación de factores de riesgo y factores protectores de la comunidad, en los encuentros talleres propuestos en concordancia con los resultados del diagnóstico se evidencia la participación activa de jóvenes y personas mayores, lo que se vuelve significativo en el desarrollo de la estrategia pedagógica, dado que las vivencias, de dos grupos poblacionales según ciclo de vida, aporta directamente a la visión de desarrollo regional. De esta manera, se promueve la aprehensión de la estrategia pedagógica, que interioriza los componentes de educación para la paz y el proceso de transmisión intergeneracional. Igualmente, este grupo heterogéneo permite analizar las afectaciones y los patrones de victimización sufridos a causa del conflicto.

- Estudio de las posiciones discursivas y de los planteamientos de los actores respecto a la problemática y a las soluciones a implementar: en esta fase se aplican técnicas de investigación cualitativa concretas, entrevistas a profundidad, grupos de discusión, entrevistas grupales, y otras de carácter participativo, como la realización de talleres con los diferentes actores, quienes a través de la aplicación de figuras propias de la administración como DOFA, flujograma, grupos nominales, entre otros, permiten analizar las afinidades y discrepancias existentes entre los actores comunitarios y conocer la percepción-explicación causal de los problemas. Así se establecen las alternativas a aplicar para solucionar dichos problemas, al igual que las responsabilidades de cada actor en el desarrollo de las acciones propias de la cultura de paz.

Plan de acción concreto: para la realización del plan de acción se hace necesaria la información que ha sido previamente recolectada a través de las fases anteriormente nombradas esto es, se tiene en cuenta, los problemas detectados inicialmente en la apreciación situacional, y los análisis de las redes y posiciones discursivas de los actores. El plan se enfoca en dos direcciones: por un lado, al apreciar las redes de los actores y sus motivaciones y estrategias, es fácil reconocer cuáles son sus propuestas y, por otro, en el momento de elaboración de los contenidos de las propuestas se jerarquiza la intervención en los 
nudos críticos de cada cadena causal. Es decir, se priorizan las propuestas y se reconocen los actores responsables en la ejecución de cada una de las actividades. En el plan aparecen los tres sectores de población fundamentales que existen en la comunidad: representantes institucionales, población formalmente organizada (asociaciones de vecinos, de empresarios, juveniles, educativas, entre otras) y base social o resto de la población que no está involucrada en ningún colectivo.

En la formulación del plan de acción, por ejemplo en una de las zonas rurales de Toluviejo, Arroyo Seco se evidencia que solo el 17 $\%$ de los pobladores, es decir, 12.493 habitantes, (Departamento Administrativo Nacional de Estadística, 2005) se reconocen a sí mismos como parte de una organización o grupo base, situación que demuestra la necesidad de generar participación activa e involucrar a las organizaciones en los distintos escenarios, de tal manera que aporten desde sus organizaciones al desarrollo social y económico de la comunidad. En el proceso de fortalecimiento realizado, se determinó que si bien existe una representación por parte de la Junta de Acción Comunal, hay aún muchos vacíos sobre los derechos a la participación que tienen como Junta de Acción Comunal- JAC en el desarrollo local y municipal, específicamente en la contribución al mejoramiento de la calidad de vida de los pobladores de su comunidad.

Vicenc Fisas (2011) ha propuesto que la construcción de paz se debe generar desde la mente de las personas participantes en los roles sociales, comunitarios, laborales, respetando los derechos humanos y las libertades fundamentales, generando el fortalecimiento de valores como la comprensión, la tolerancia, la amistad entre las naciones, todos los grupos raciales y religiosos.

- Paso a la acción: el paso a la ejecución de lo planteado previamente requerirá la capacidad de reconducir las situaciones nuevas que se presenten, monitorear las dificultades no previstas y tener la posibilidad de evaluar y corregir constantemente los procesos. Para esto hay que dotarse de una serie de instrumentos y técnicas organizativas capaces de afrontar un cronograma, que se comporte como un documento dinámico capaz de afrontar las diferentes coyunturas que 
se presenten. Con esta intención se establecerán mecanismos de evaluación que están enfocados en el cronograma y organigrama de funcionamiento, así como momentos de revisión para que quede acorde con los requerimientos de la población.

\section{Diagnóstico rural participativo- DRP}

El diagnóstico rural participativo -DRP es un conjunto de técnicas y he- rramientas que permite que las comunidades rurales hagan su propio diag- nóstico y a partir de este a auto-gestionar su planificación y desarrollo. De esta manera, los y las participantes podrán compartir experiencias y anali- zar conocimientos propios y ajenos, a fin de mejorar sus habilidades de planificación y acción.

El DRP pretende desarrollar procesos de investigación desde las condiciones y posibilidades del grupo meta. Para este análisis, se define como fundamental analizar los conceptos e imaginarios que se conciben como propios. Generalmente, las investigaciones plantean al inicio una cantidad de preguntas que están formuladas con anterioridad; sin embargo, dado los ejes de la educación para la paz, se concibe la idea de que la población misma realice un diagnóstico. La intervención de las personas que componen el equipo que facilita el DRP debe ser mínima; idealmente se reduce a poner a disposición las herramientas metodológicas de educación para la paz para el autoanálisis de los participantes y retirarse para dejar la interacción en manos de la población en sí. Cabe resaltar que no se pretende únicamente recoger datos del grupo meta, sino que este inicie un proceso de autor-reflexión sobre sus propios problemas y encuentre las posibilidades de solución desde una actitud pacifista.

El objetivo principal del DRP es apoyar la autodeterminación de la comunidad rural a través de la participación y así fomentar un desarrollo sostenible, sin que a largo plazo se vean afectados ninguno de los dos sujetos participantes, en este caso la naturaleza (territorio) y las personas víctimas del conflicto en su rol comunitario; es en parte una medida para promover la importancia de considerar sujeto a la naturaleza y no solo objeto de explotación. Entre las principales características del DRP están: 
- $\quad$ Respeto a la sabiduría y la cultura del grupo meta

El respeto al conocimiento cultural en el manejo de los recursos naturales y la identidad por el arraigo es fundamental para las aplicaciones estratégicas de educación para la paz, ya que el desarraigo fue consecuencia de lo acontecido por la presencia del actor armado en el territorio. La identidad cultural, valores, normas, visiones, conocimientos y costumbres, produce vías endógenas de desarrollo particular para cada comunidad. La función del equipo profesional es escuchar y enseñar a partir de los conocimientos previos. En palabras de Francisco Jiménez Bautista (2011) en su texto Racionalidad pacífica. Una introducción a los estudios para la paz, "se trataría de tener una visión objetiva, crítica, que no se quede en los elementos negativos que tiene nuestra sociedad. Aportar soluciones, alternativas, ideas frescas, en definitiva, ser < expertos> de una educación para la paz" (p. 120).

El enfoque propuesto por Hicks en el texto Educación para la par. (1993) centra el análisis del conflicto desde lo personal a lo global y desde la manera de resolverlo sin violencia. Lo anterior se puede entender como un reflejo de la comunidad, consecuencia de las conflictividades vividas en épocas álgidas del conflicto armado interno en Montes de María y la capacidad de resolver el conflicto de manera pacífica.

- Análisis y comprensión de las diferentes percepciones

Cada sujeto y cada cultura perciben la realidad de forma subjetiva y propia, sin que esta interpretación deje de tener validez. Los miembros de las comunidades, los promotores en el campo del desarrollo y los investigadores generalmente observan e interpretan el mundo en el que viven de diferentes maneras. A través de un proceso de comunicación y un aprendizaje mutuo se pueden vislumbrar diferentes percepciones, a tal punto de que los actores se comprendan y actúen conjuntamente; sin embargo, es necesario un proceso de aprendizaje mutuo entre la población rural y el equipo investigador. Las diferentes percepciones conducen a contradicciones en la aplicación e interpretación de algunos instrumentos del DRP, que a su vez son la base del aprendizaje complejo, característica clave de este tipo de investigación. 
Resulta esencial involucrar y dirigirse en particular a aquellas partes de un grupo o una comunidad que normalmente no son escuchadas, sea por la falta de elocuencia, porque son iletrados o por una posición social marginal. La propuesta pedagógica propone trabajar en grupos homogéneos, es decir, con personas pertenecientes a un mismo sector social, y ayudarse a través de la utilización de medios de visualización, motivando especialmente a las personas que se mantienen al margen del proceso de comunicación. Se reconoce que un motivante para la participación activa de personas que hacen poco uso de la palabra es sentirse acompañados por su "grupo social", así encuentran en el contexto la posibilidad de expresarse libremente, sin sentirse intimidadas, y adquieren cada vez más el empoderamiento y los lazos de confianza que permiten una capacidad de escucha superior y una vivencia más pacífica, reconociendo a todos como sujetos de derechos.

\section{Pedagogía transformadora}

El centro de construcción pedagógica que se propone es la persona y su desarrollo a través del proyecto de vida. Se parte de una concepción diná- mica del ser humano en el encuentro con el otro, quien descubre un hori- zonte común y trascedente a su existencia. Se encuentra, en este sentido, gran sintonía con los planteamientos fundamentales del personalismo y su apuesta decidida en favor de la integridad de la persona. De esta manera, se reconoce una actividad fundamental en la vida del ser humano: el tra- bajo, el cual se concibe como, más que una obligación, una necesidad fun- damental para humanizar y descubrir la solidaridad, y finalmente para pro- mover la vocación personal y social, su fidelidad y responsabilidad con la comunidad y el territorio que se comparte.

$\mathrm{Al}$ reconocer de gran utilidad para el desarrollo de este tipo de conceptos la corriente de pensamiento del personalismo, se concibe la libertad en oposición al miedo de vivir. Su seguridad está basada en la paciencia y la confianza, y adquiere en el sufrimiento un valor fundamental porque este es superado en un don de sí que es virtud y fortaleza, y la contemplación es la cumbre de esta lucha incesante consigo mismo, con la naturaleza y con los demás. La finalidad de alcanzar una meta es la visión de un ideal en común. Como afirma Chávez, "para los personalistas, el hombre de 
hecho, desde que nace es una persona y posee, por tanto, una subjetividad libre, lo que no niega la necesidad de la socialización, para alcanzar la plena dimensión como persona" (1997, p.31).

Algunos de los aspectos relevantes para la pedagogía transformadora son:

- $\quad$ Esperar con toda la esperanza: En comunidades victimizadas por la violencia, recobrar la confianza entre los actores que comparten el territorio, se convierte en un desafío. De manera inherente a la vida del ser humano se encuentran momentos de crisis en los cuales la situación parece estar perdida y la salida no se evidencia de manera clara. Dichas crisis tienen a su vez una parte positiva, pues motivan a buscar alternativas que permiten el crecimiento. En ese instante, el ser humano es capaz de rebelarse contra el dolor y contra la adversidad, y tomar recursos externos e internos para salvarse. "Montes de María cree en la vida a pesar de la muerte, porque la vida es más fuerte que la muerte" (Castillo Torres, 2009: 15).

- Junto a otros se construye la esperanza: Según Torres (2008, p.10), hacernos humanos y humanas en la interacción, la vincularidad y el mutuo reconocimiento es tarea de todos los días y de cada día. Por ello se habla de cotidianidad educativa o de día en la construcción de lo humano, reconocernos como sujetos y sujetas de derecho, con deberes comunes para el desarrollo humano, social, cultural y regional. Desde el ámbito de la educación, deconstruir la violencia cultural es fundamental en el desarrollo de la educación para la paz en comunidades víctimas de violencia armada.

- Lo más concreto, lo más conocido y lo más próximo: Ahí donde se entretejen las formas de la sobrevivencia, la resistencia y la esperanza, ahí mismo se acortan las alas de acción educativa y se presenta la oportunidad para resituarla frente al desafío de la lucha permanente por la vida y por el sentido de la vida, denotando la transformación en la cotidianidad de los conflictos, propios de la comunidad resueltos ahora de manera no violenta.

- El lenguaje positivo: El vocabulario tiene la capacidad de permear el ambiente de manera propicia para la construcción de la 
cultura de paz y generar redes de esperanza y confianza; así, la palabra es una de las muchas formas de renacer. Conocer la historia permite recrear momentos pasados y hablar del presente; en otras palabras, renacer. Fortalecer la empatía, la solidaridad, el respeto por las diferencias, la equidad, la dignidad y el amor son maneras de formar seres resilientes y aumentar la capacidad de convertir las crisis en oportunidades, de crear vida y formas de vidas sostenibles para toda persona, para los otros y para las generaciones que proceden. Pensarse a sí mismo de forma positiva y verbalizar los puntos positivos que cada persona tiene y que tienen los demás afina los vínculos y fomenta el buen trato y las palabras de reconocimiento y el respeto por el otro.

\section{Resiliencia}

El vocablo resiliencia tiene su origen en el idioma Latín, en el término resalió que significa volver atrás, volver de un salto, rebotar, el término fue adaptado a las ciencias sociales para caracterizar aquellos sujetos que a pesar de nacer y vivir en situaciones de alto riesgo, se desarrollan psicológicamente sanas y socialmente exitosos (Villalba, 2004, p.32)

Julián Reid y Brad Evans (2016), por su parte, en su texto Una vida en resiliencia: el arte de vivir en peligro plantean el concepto de resiliencia, basándose inicialmente en el antropocentrismo y la capacidad que tiene el ser humano por "ley natural de eliminar al más débil" y destrozar a aquel que se interpone en la consecución de sus intereses como una forma normal de evolución y desarrollo. Se evidencia en el análisis de estos autores las formas políticas en las que se institucionaliza y se justifica la violencia, y se da reconocimiento a la persona que se considera resiliente. Para el presente caso práctico es importante mencionar que la resiliencia que se busca incentivar en la población está más allá del perdón es la capacidad misma de recordar y asumir los sucesos como situaciones que en el marco de la construcción de paz no deben repetirse y que en el caso de darse repetición, se deben encontrar las formas de contrarrestar el daño y realizar acciones propias desde los ejes de educación para la paz, con enfoque de derechos aprehendidos en la comunidad.

Por tanto, la resiliencia se considera una capacidad esencialmente humana y universal que involucra al ser humano por completo e incluye su 
espiritualidad, sus sentimientos, sus experiencias, acciones y cogniciones, que son determinantes en el desarrollo de las personas, desde edades tempranas. En conclusión, hay una gran variedad de respuestas individuales frente a los eventos y circunstancias adversas, vivencias que se convierten en las claves para la transformación pacífica del conflicto y conducen al fortalecimiento del ser humano y de la comunidad desde la solución de las situaciones problémicas hasta la construcción de sus propuestas pacíficas.

Este proceso se realiza a través del tiempo y presenta afortunadas combinaciones entre los atributos de la persona y su ambiente familiar, social, comunitario y cultural. Así, la resiliencia no puede ser pensada como un atributo con que se nace o se adquiere en el desarrollo, sino que se trata de un proceso que caracteriza un complejo sistema social, en un momento determinado del tiempo (Rutter, 1993), y tiene en cuenta los factores protectores, esto es, las condiciones o los entornos capaces de favorecer el desarrollo de individuos o grupos según su ciclo vital y, en muchos casos, de reducir los efectos de circunstancias desfavorables, en busca de la transformación pacífica de los factores de riesgo. Se constituyen como moderadores e incluyen tanto recursos personales como sociales, y se pueden distinguir entre externos e internos. Los externos se refieren a condiciones del medio en que actúan reduciendo la posibilidad de daños: familia extendida, apoyo de un adulto significativo o integración social y laboral. Los internos se refieren a atributos de la propia persona: estima, seguridad y confianza en sí mismo, facilidad para comunicarse y empatía.

\section{Educación popular}

La educación popular es una corriente político-educativa construida his- tórica y contextualmente en Latinoamérica, es decir, tiene una identidad propia marcada por una realidad histórica y sociopolítica. Se puede hablar de un momento fundacional de la educación popular a finales de los se- tenta, cuando se convirtió en un discurso educativo y en una corriente colectiva, cuyo inmediato y principal expositor es Paulo Freire, pedagogo brasileño. La educación popular se alimenta simultáneamente de varias di- mensiones de la realidad: referentes teóricos previos, imaginarios colecti- vos, representaciones y valores culturales, y experiencias compartidas, así como de la reflexión sobre las propias prácticas educativas. La educación 
popular se va configurando y redefiniendo permanentemente tanto en sus presupuestos como en sus prácticas, atendiendo a la dinámica propia de la zona rural donde se tome como referente conceptual.

\section{Resultados y conclusión}

La implementación de la educación para la paz en comunidades víctimas de la violencia armada requiere un abordaje desde múltiples dimensiones. Así el diagnostico en primera medida construido desde la misma comuni- dad, flexibilizará y amoldará las estrategias a utilizar. Tal lo evidencia el análisis de los resultados de los encuentros grupales, donde se aplican los preceptos de educación para la paz con un enfoque de derechos humanos.

El aporte a la cultura política es un cambio temprano evidenciado en las comunidades, que infiere en la participación comunitaria de visión de desarrollo en el territorio, el auto reconocimiento como sujetos políticos y sujetas políticas a partir del aprendizaje de derechos humanos y sus roles en la comunidad como factor importante de relación con la empresa pri- vada y otros actores comunitarios. Se pudo constatar este cambio signifi- cativo, de cara a la incidencia que los lugareños tuvieron en los planes de desarrollo locales atendiendo a la hoja de ruta para el desarrollo regional.

La educación para una cultura de paz, en la actualidad, es una educación para la vida, que implica la convivencia pacífica en la comunidad, aprendiendo a respetar el marco ético de los derechos humanos y la relación con la naturaleza, entendida esta en el sentido amplio de arraigo territorial y cultural. La preparación territorial para escenarios de posacuerdos de paz y la relación empresa privada - comunidad son rasgos característicos de estrategias de educación para la paz, aprehendidas en comunidades victimizadas por la violencia armada. En las cuales se utilizaron estrategias completamente participativas y comprensibles según sus capacidades de entendimiento, logrando generar inquietudes en las personas participantes, lo que va desglosando el acuerdo de paz, que en los encuentros talleres fue tema imperativo, dada la coyuntura del país entre 2014 y 2015.

La responsabilidad social por la paz implica hacer reflexiones constantes en la metodología utilizada en comunidades víctimas de la violencia armada. Por tanto, la flexibilidad de la estrategia pedagógica Reconstruyendo 
el tejido social: una apuesta a la participación comunitaria es completamente acorde con los propósitos de empoderar a las comunidades víctimas de violencia armada en Montes de María. Los postulados del Programa de Desarrollo y Paz de los Montes de María, en su rol articulador dentro del territorito con aliados estratégicos, siguen presentando gran relevancia para la llegada a comunidades victimizadas.

El compromiso de la educación para la paz no debe existir solo en la teoría, se construye día a día con los actores sociales con presencia en el territorio, desde sus distintos roles comunitarios, siempre apostando a una convivencia pacífica y a soluciones alternativas, que nacen del diálogo, el respeto mutuo y la identidad cultural en espacios protectores como la comunidad.

Es importante tener en cuenta al momento de educar para la paz en comunidades victimizadas el aspecto cognitivo del grupo meta según su ciclo de vida, ya que la percepción de la violencia y el conflicto cambia. Se debe volver significativa la participación en la dinámica comunitaria, en la cual se puedan plantear y buscar soluciones eficaces a problemáticas cotidianas, donde prime el respeto a los derechos humanos, las libertades de los semejantes, el cuidado al medio ambiente, lo que fortalecerá un enfoque humanístico, el cual permite el aprendizaje significativo desde el ser y el saber hacer.

Educar para la paz en comunidades víctimas del conflicto significa sentar las bases en intercambio generacional, teniendo como eje los valores de justicia y solidaridad, lo que incluye la construcción del proyecto de vida en heterogeneidad, teniendo como base preceptos propuestos por Fisas (2011, p.5) aprender a conocer, aprender a hacer, aprender a vivir juntos, aprender a ser. Así, se presentarán escenarios para la reconciliación y superación de conflictividades cotidianas.

El reconocimiento del territorio, su cultura e idiosincrasia es para la educación para la paz parte del proceso transformador en clave pacífica. Un hallazgo relevante de las comunidades es la comprensión y aceptación de la empresa privada con presencia en el territorio. Reconoce la importancia de esta como un aliado estratégico en la garantía de derechos fundamentales y cuidado del entorno, categorías que presentan, para efectos 
de este estudio, un logro significativo en cuanto a seguridad de la comunidad y estabilidad de la empresa privada.

De acuerdo con Jean Paul Lederach (2000), la transformación de conflictos en comunidades víctimas de la violencia implica percibir y responder a fluctuaciones de los conflictos sociales como una oportunidad para crear procesos de cambio constructivos que reduzcan la violencia.

Así desde la pedagogía para la paz, en comunidades que han pasado episodios largos de violencia armada, se convierte en eje fundante de una mejor convivencia y en preparación de escenarios de desarrollo territorial desde distintos ejes: políticos, sociales, culturales, económicos y de goce efectivo de derechos. La flexibilidad de estrategias pedagógicas para la paz, debe contribuir al aprendizaje desde los saberes intrínsecos de las personas que comparten un horizonte de futuro, garantizando que la aprehensión de los conocimientos básicos propuestos se pueda desarrollar respetando el auto proceso de aprendizaje.

El monitoreo y revisión constante de las estrategias de educación para la paz implica el aprendizaje de ser miembros de una comunidad global, en el sentido de la convivencia armoniosa y la resolución pacífica de conflictos, sentar estas bases significa preparar a las nuevas generaciones y aportar a los espacios de encuentros culturales que para comunidades victimas en Montes de María ha sido la manera más fehaciente de resistencia.

\section{Referencias}

Camacho, J., \& Silva, N. (2013). Concentracion, acaparamiento de tierras, desarrollo rural $y$ derecho a la alimentacion. Bogota: U. Externado de Colombia. Recuperado el 10 de Julio de 2018

Castillo Torres, R. (2009). Espiritualidad montemariana. Cartagena: Departamento de Comunicaciones Fundación Red Desarrollo y Paz de los Montes de María.

Colombia.co. (2013). ¿Cómo es la organizacion politico-administrativo de Colombia? Obtenido de http://www.colombia.co/esta-es-colombia/estructura-delestado/como-es-la-organizacion-politico-administrativa-de-colombia/

Constitución Política de Colombia. (1991). Bogotá. 
Departamento Administrativo Nacional de Estadística. (2005). Proyecciones 20052020.

Fisas, V. (2011). Educar para una cultura de paz. Quaderns de construccio de pau(20). Freire, P. (s.f.). Pedagogía del oprimido.

Fundación Red Desarrollo y Paz Montes de María. (2015). Diagnóstico socioeconómico. Municipio de Tolú Viejo. Cabecera Municipal: Tolú Viejo. Corregimiento: La Piche. Veredas: La Granja-Arroyo Seco-Cienaguita. Fundación Red Desarrollo y Paz de los Montes de Maria, Sucre. Sincelejo: Equipo de comunicaciones FRDPMMa.

Galtug, J. (2004). Violencia, guerra y su impacto sobre los efectos visibles e invisibles de la violencia. Obtenido de http://red.pucp.edu.pe/wpcontent/uploads/biblioteca/081020.pdf

Galtung, J. (2003). Pazpor medios pacíficos. Guernica y Luno: Gernika Gogoratuz.

Grupo de Memoria Historica. GMH. (2013). ¡Basta Ya! Colombia: Memorias de guerra y dignidad. Bogota: Imprenta Nacional.

Hicks, D. (1993). Educación para la pa\%: Madrid: Ediciones Morata.

Jiménez Bautista, F. (2011). Racionalidad pacifica. Una introducción a los estudios para la par:

Lederach, J. P. (2000). El abecé de la pazy los confictos. Educación para la paz: Madrid: Catarata.

M, F. T. (2008). Nueva Tierra.

Mendoza, E. P. (2010). Los Montes de María: Análisis de la conflictividad. Programa de las Naciones Unidas para el Desarrollo -PNUD- Montes de María. New York: Impresol.

Muñoz, F. (2001). La paz imperfecta en un universo en conflicto. En F. A. Muñoz, La paz imperfecta (págs. 21 - 66). Granada: (ed).

PNUD. (2010). Análisis del desarrollo relacionado con el conflicto.

Reid, J., \& Evans, B. (2016). Una vida en resiliencia: el arte de vivir en peligro.

rodriguez, j. c. (1997). Filosofia de la educacion superacion para el docente.

Salazar, H. (2014). Conflicto interno y paz en Colombia: visibilización desde la academia. Cartagena: U.D.C.A.

Sandoval Forero, A. (2015). Empoderamiento pacifista para otros mundos posibles. Revista de Paz, y Conflictos, 8(2), 75-95. Obtenido de https://www.ugr.es/ revpaz/numeros/revpaz_8_2_completo.pdf 
Terry Gregorio, J. R. (Diciembre de 2007). Teoría y práctica del desarrollo comunitario rural integrado. Revista OIDLES, 1(2). Obtenido de http://www.eumed.net/rev/oidles/02/Gregorio.htm

Unidad para las Víctimas. (2018). Registro Único de Victimas (RUV). Obtenido de http://www.unidadvictimas.gov.co/es/registro-unico-de-victimas$\operatorname{ruv} / 37394$ 